\title{
Fictional narratives change beliefs: Replications of Prentice, Gerrig, and Bailis (1997) with mixed corroboration
}

\author{
S. CHRISTIAN WHEELER, MELANIE C. GREEN, and TIMOTHY C. BROCK \\ Ohio State University, Columbus, Ohio
}

\begin{abstract}
We report three exact replications of experiments aimed at illuminating how fictional narratives influence beliefs (Prentice, Gerrig, \& Bailis, 1997). Students read fictional stories that contained weak, unsupported assertions and which took place either at their home school or at an away school. Prentice et al. found that students were influenced to accept the assertions, even those blatantly false, but that this effect on beliefs was limited to the away-school setting. We questioned the limiting of the narrative effect to remote settings. Our studies consistently reproduced the first finding, heightened acceptance of statements occurring in the conversations of narrative protagonists, but we failed to reproduce the moderating effect of school location. In an attempt to understand these discrepancies, we measured likely moderating factors such as readers' need for cognition and their extent of scrutiny of the narratives.
\end{abstract}

The present article reports exact replications of recent experiments aimed at illuminating how fictional narratives may influence beliefs (Prentice, Gerrig, \& Bailis, 1997). Prentice et al. showed that acceptance of statements, even ones that were clearly false, can be induced by presenting the statements as assertions in narrative dialogues. The effect was limited, however, to remote (unfamiliar), rather than local (familiar), story settings. The moderating effect of story setting prompted Prentice et al. to propose likely mediating mechanisms such as forms of cognitive scrutiny that would presumably be more readily activated for familiar settings than for unfamiliar settings. By measuring several of these potential moderators, we sought to corroborate and explain the Prentice et al. findings.

Does use of familiar elements in fact limit the impact of fictional narratives? To us, it seemed that fiction often achieves verisimilitude by exploitation of familiar elements such as local settings, famous personages, and commonly known events. Our questioning of the alleged limiting impact of familiarity stemmed in part from consideration of the power of fictional narratives, both negative and positive, on national and cultural levels. From the parables of Jesus to the lyrics of contemporary rock music, the power of fiction has not appeared to be limited to narratives about remote places or eras.

We are indebted to Deborah Prentice for assisting in our exact replications of her studies; she provided her materials and details of her experimental procedures, as well as helpful comments on preliminary drafts of this report. We thank Jennifer Garst for her comments. This study was supported by Grant T32-MH 19728 from the National Institute of Mental Health. Melanie C. Green was a National Science Foundation Graduate Fellow during completion of this work. Correspondence should be addressed to T. C. Brock, Department of Psychology, Ohio State University, Columbus, OH 43210-1222 (e-mail: brock.1@osu.edu).
We were also motivated to reproduce the Prentice et al. (1997) results because it is ordinarily difficult to gain agreement with false statements (Eagly \& Chaiken, 1993). Yet Prentice et al. found that blatantly false statements (e.g., "Eating chocolate makes you lose weight" and "Most forms of mental illness are contagious") could change beliefs when these statements occurred as parts of conversation in a fictional narrative.

In our investigation of moderators of the Prentice et al. (1997) effect, we were guided by the theoretical perspectives of dual-processing models of persuasion (e.g., Chaiken, 1987; Petty \& Cacioppo, 1986). These models predict that when individuals are either unmotivated or unable to scrutinize weak arguments they may rely on cues or heuristics (e.g., "Authors of fiction don't just make things up"; Prentice \& Gerrig, in press) to determine belief change. When requisite motivation and ability are present, however, elaborative processing should result in the rejection of weakly supported assertions. Thus, factors promoting elaboration, such as personal relevance or the propensity to engage in effortful thought (need for cognition, NC; Cacioppo, Petty, \& Kao, 1984), should moderate persuasion by weak arguments.

Prentice et al. (1997) adopted Gilbert's (1991) proposal that people involuntarily believe text they understand and only later discount it due to corrective information. Thus, individuals would be automatically persuaded by the assertions in narratives unless factors are present that elicit scrutiny. In contrast to Samuel Taylor Coleridge's "willing suspension of disbelief," Prentice et al. proposed a willing construction of disbelief. According to this hypothesis, readers must actively work towards refuting story assertions and will do so if the story setting is familiar. However, if an unfamiliar setting precludes such special effort, readers will accept story assertions as true. 
To test the story-distance hypothesis, Prentice et al. (1997) wrote two versions of a story, one of which took place at the subjects' own college and one of which took place at a distant college. During the story, the characters made assertions about real-world information. Half of the assertions were true and half were false. All assertions were weakly supported and should not have withstood careful scrutiny.

When the story took place at a distant setting, subjects were persuaded by the story assertions, relative to control subjects. When the story took place at the subjects' own college, however, the subjects were not reliably persuaded by the assertions. We attempted to reproduce the striking impact of narrative assertions on beliefs and to verify that this impact was indeed limited to narratives that occurred in less familiar settings. We sought to increase understanding of underlying mechanisms by measuring selected moderating variables such as Cacioppo et al.'s (1984) NC.

\section{EXPERIMENT 1}

\section{Method \\ Subjects. The subjects were undergraduates $(N=220)$ at Ohio State University who were compensated with partial course credit. Sixty-three of them read the control story and 157 of them read one of the experimental stories.}

Procedure. The design was adapted from Prentice et al. (1997). Experimental subjects read a story that contained both true and false assertions about real-world topics. The stories took place either at Yale or at Ohio State University (OSU). The Yale story was the same as that used by Prentice et al.; the OSU story was simply the Yale story with the setting elements changed. During the course of the story, the characters discussed 15 real-world topics. ${ }^{1}$ Half (i.e., seven or eight) of the assertions made by the characters were true, and half were false. The veracity of story assertions was counterbalanced within each story version. The stories were approximately 20 single-spaced pages long.

Control subjects read "The Raven," by Robert Twohy, the same control story used by Prentice et al. (1997). This story contained no assertions relevant to the dependent measures and thus permitted the beliefs of the control subjects to serve as a proxy for the preexperimental baseline beliefs of subjects in the experimental conditions.

After finishing the story, the subjects rated the quality of the stories and then completed one of two intervening tasks. Two-thirds of the subjects completed a self- and other-description questionnaire, as in the original Prentice et al. (1997) design. The other third of the subjects completed a portion of the verbal SAT test and the $\mathrm{NC}$ scale (Cacioppo et al, 1984). The NC scale is widely used to assess the degree to which an individual enjoys thinking (Cacioppo, Petty, Feinstein, \& Jarvis, 1996). Both intervening tasks took approximately 12 min.

Following the intervening tasks, the subjects filled out a questionnaire that assessed their level of agreement with 32 statements. For each test item, the subjects rated their level of agreement with the statement on a 9-point Likert scale anchored by 1 (completely disagree) and 9 (completely agree). Fifteen of 32 items corresponded to assertions made by the characters in the story. Half of the items (i.e., either 7 or 8 ) were congruent with the assertions made in the story and half of them were incongruent with story assertions. In addition, half of the items were true and half were false. There were two versions of the questionnaire, which counterbalanced the truth status of the test items. Thus, as in the original Prentice et al. (1997) experiments, the test items and story assertions were crossed in a 2 (story assertion: true or false) $\times 2$ (test item: true or false) within-subjects design. Finally, the subjects com- pleted a 9-item recall test of basic information from the story, such as, "What was the name of the main character?" (Wheeler, 1997).

\section{Results and Discussion}

Ratings of message quality and recall scores were uninfluenced by any experimental factors, and therefore these measures are not further discussed.

According to the hypothesis and the past research of Prentice and her colleagues, subjects who had read the story that took place at another school (Yale) should have been more persuaded by the story assertions than should the subjects who read the story that took place at their own school (OSU). To conduct these analyses, the mean agreement ratings of the control subjects for true and false test items were subtracted from the agreement ratings of each subject for each true and false test item, respectively. Thus, subjects who agreed with test items less than the control subjects would have a negative score, while subjects who agreed more than the control subjects on a given test item would have a positive score.

If subjects were influenced by the story assertions, they should differ from control subjects in their agreement with the test items. Specifically, subjects who were persuaded by the story assertions should show more agreement than would the control subjects when the story assertion and test item were congruent (i.e., both true or both false) and less agreement than control subjects when the story assertion and test item were incongruent. For instance, subjects who read false story assertions should report more agreement with false test items than with true test items. Hence, throughout this article, an interaction between story assertion and test item indicates persuasion by the story assertions. If story location moderated these results, the interaction would be observed only in the away-school version. Thus, a three-way interaction between location, story assertion, and test item should emerge. Means for these comparisons are presented in the top panel of Table 1.

The means indicated that subjects were influenced by the story assertions across story-location conditions. Subjects agreed with the test items less when the story assertions were incongruent with the test item than when they were congruent, as evidenced by a reliable story assertion $\times$ test item interaction $\left[F(1,152)=78.28, M S_{\mathrm{e}}=\right.$ $1.12, p<.0011$. If this effect was moderated by the story location, these results should have been qualified by a three-way interaction between location, story assertion, and test item. This interaction did not attain significance $\left[F(1,152)=1.49, M S_{\mathrm{e}}=1.12, p<.25\right]$. In contrast to the results obtained by Prentice et al. (1997), we obtained significant two-way interactions for both the OSU $[F(1,79)$ $\left.=44.10, M S_{\mathrm{e}}=1.12, p<.001\right]$ and the Yale $[F(1,73)=$ $\left.35.53, M S_{\mathrm{e}}=1.12, p<.001\right]$ story conditions.

Prentice et al. (1997) reported summary graphs showing average persuasion in the direction of the story assertions. Their graphs are reproduced as the two leftmost graphs in Figure 1. Although we obtained strong evidence for the impact of assertions on beliefs in Experiment 1, 
Table 1

Agreement Ratings for Target Items Relative to Control Subjects

\begin{tabular}{|c|c|c|c|c|c|c|}
\hline \multirow[b]{2}{*}{ Test Item } & \multicolumn{2}{|c|}{$\begin{array}{l}\text { Home-School (OSU) Story } \\
\text { Asserted in Story }\end{array}$} & \multicolumn{2}{|c|}{$\begin{array}{c}\text { Away-School (Yale) Story } \\
\text { Asserted in Story }\end{array}$} & \multicolumn{2}{|c|}{$\begin{array}{l}\text { Combined Means } \\
\text { Asserted in Story }\end{array}$} \\
\hline & True & False & True & False & True & False \\
\hline \multicolumn{7}{|c|}{ Experiment 1} \\
\hline True & .08 & -.90 & .01 & -.71 & .05 & -.81 \\
\hline False & -.33 & .70 & -.26 & .56 & -.30 & .63 \\
\hline \multicolumn{7}{|c|}{ Experiment 2} \\
\hline True & .01 & -.29 & .35 & -.32 & .18 & -.31 \\
\hline False & .19 & .81 & -.11 & .64 & .03 & .72 \\
\hline \multicolumn{7}{|c|}{ Experiment 3} \\
\hline True & & -.77 & & -.49 & & -.63 \\
\hline False & & .55 & & .63 & & .59 \\
\hline
\end{tabular}

we were unable to repeat the story setting effect (center graph in Figure 1).

We conducted additional analyses for the subset of subjects that had the NC scale as an intervening task. In the analysis of variance (ANOVA), a median split was conducted to distinguish between persons who were high in $\mathrm{NC}$ (HNC individuals) from persons who were low in $\mathrm{NC}$ (LNC individuals; median $=62$, theoretical range $=$ 18-90, actual range $=30-84)$. We might expect $\mathrm{NC}$ to interact with location, story assertion, and test item such that $\mathrm{LNC}$ individuals reading the away-school story would show higher levels of persuasion than LNC individuals reading the home-school story. Such individuals would be unlikely to elaborate upon the story information unless situational factors (here, story location) prompted greater cognitive effort. In contrast, $\mathrm{HNC}$ individuals are likely to engage in cognitive elaboration regardless of the situation, and thus should reject the weakly supported arguments in both stories. Thus, NC may moderate the location effect, and the focal four-way interaction could reveal the location effect for a subset of our subjects (i.e., LNC subjects).

Within the subset of subjects that completed the $\mathrm{NC}$ scale as an intervening task $(N=50)$, individuals were highly influenced by the story in a pattern similar to the full sample [story assertion $\times$ test item, $F(1,46)=48.39$,

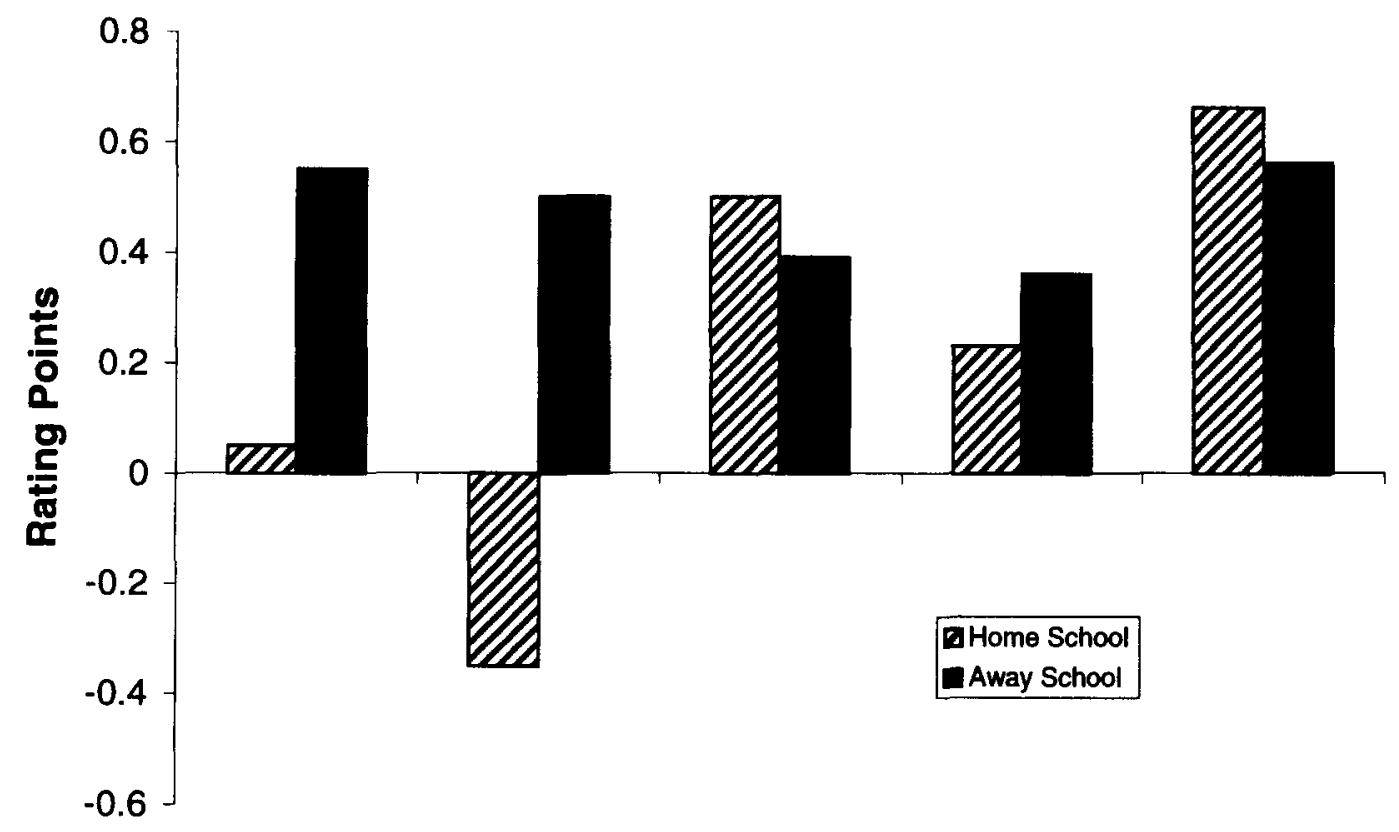

Prentice et al., Prentice et al., Replication 1 Replication 2 Replication 3 Exp. $1 \quad$ Exp. 2

\section{Experiment}

Figure 1. Average persuasion in the direction of story assertions from Prentice et al. (1997) experiments ( 1 and 2 ) and present replications (1, 2, and 3). 
$\left.M S_{\mathrm{e}}=1.12, p<.001\right]$. However, the focal NC $\times$ location $\times$ story assertion $\times$ test item interaction failed to achieve significance $\left[F(1,46)=.96, M S_{\mathrm{e}}=1.12, p<.35\right]$, suggesting that persuasion among $\mathrm{HNC}$ and LNC subjects did not differ by location. ${ }^{2}$

One possible reason for our failure to replicate the Prentice et al. (1997) story-location effect was that our manipulation of location did not affect perceived personal relevance-the instigation to engage in critical scrutiny. We therefore conducted a supplementary study using an additional sample from the same subject pool. Forty subjects read one of the three stories (OSU, Yale, or control) and responded to the question, "How relevant is the story to you?" on a 5-point Likert scale. A planned contrast indicated that the OSU story was considered more personally relevant $(M=3.29)$ than both the Yale $(M=2.13)$ and the control $(M=2.38)$ stories $\left[t(1,37)=2.33, M S_{\mathrm{e}}=.45, p<\right.$ $.05]$. These data buttressed the Prentice et al. assumption that a local story setting would elicit more felt personal relevance than a remote story setting. However, the data also raised a question about the impact of personal relevance on the processing of narrative fiction. Given that the absence of a story-location effect was not due to failure to induce differences in perceived personal relevance, was the absence perhaps due to the failure of the manipulation of story setting to produce differences in scrutiny of the message? To evaluate this possibility, we included a direct measure of such scrutiny in the next experiment.

\section{EXPERIMENT 2}

This experiment attempted again to replicate the pattern of results obtained by Prentice et al. (1997). In addition, it included a measure (Green, 1996) designed to assess the amount of scrutiny applied to a narrative passage.

\begin{abstract}
Method
Subjects. Students $(N=68)$ from Ohio State University participated as subjects in Experiment 2 for partial course credit. Twenty subjects read the control story and 19 subjects read each of the experimental stories.

Design and Procedure. The materials and procedure remained the same as in Experiment 1 with two modifications. First, in Experiment 2, only one intervening task was used: the $\mathrm{NC}$ scale and a portion of the verbal SAT. Second, we introduced a task designed to assess the amount of scrutiny: the subjects were instructed to go through the text a second time and to circle all of the "Pinocchio's" or false-sounding portions of the text (Green, 1996). This technique provided an assessment of the amount of questioning and doubting that may have occurred while the subjects were reading the story. The Pinocchio task was administered last in the experimental procedure.
\end{abstract}

\section{Results and Discussion}

An ANOVA of subjects' beliefs revealed that subjects were again influenced by the story assertions. The story assertion $\times$ test item interaction was significant $[F(1,33)$ $\left.=6.33, M S_{\mathrm{e}}=1.38, p<.05\right]$. This interaction was not qualified by a three-way location $\times$ story assertion $\times$ test item interaction $\left[F(1,33)=.05, M S_{\mathrm{e}}=1.38, p>.80\right]$. Individual two-way interactions indicated that subjects tended to be persuaded by both the $\operatorname{OSU}[F(1,16)=2.32$, $\left.M S_{\mathrm{e}}=1.38, p<.15\right]$ and the Yale $\left[F(1,17)=4.37, M S_{\mathrm{e}}=\right.$ $1.38, p=.05]$ stories. Thus, the data (middle panel of Table 1) showed again that subjects' beliefs were influenced by the story but that the extent of influence was independent of the location of the story. Average persuasion in the direction of story assertions for Experiment 2 has been plotted in the second of the three rightmost graphs in Figure 1. As can be seen, the story-location effect from Prentice et al. (1997) was not corroborated here.

To examine the effects of NC, we conducted a median split (median $=62.5$, range $=28-88$ ). The focal four-way $\mathrm{NC}$ interaction did not attain significance $[F(1,33)=$ $\left..48, M S_{\mathrm{e}}=1.38, p<.50\right]$. Thus, as in Experiment 1, NC did not moderate the story location effect.

The Pinocchio-measure results provided some trends in support of the hypothesis that individuals were scrutinizing the information to a greater extent for homeschool story location. The Pinocchio data were coded in terms of both number of lines of text circled (identified) and total number of circles. Subjects who read the homeschool story tended to indicate more total circles $(M=$ 21.7) and more lines of text $(M=42.3)$ than did subjects reading the away-school story $(M s=16.1$ and 32.3 , respectively). However, these trends were not reliable $(p \mathrm{~s}>.15)$. Similarly, HNC individuals made more total circles $(M=19.2)$ and circled more lines of text $(M=$ $44.1)$ than did LNC subjects $(M \mathrm{~s}=18.2$ and 27.0 , respectively). The latter effect of NC on "false-noting" of lines was reliable $\left[F(1,33)=3.99, M S_{\mathrm{e}}=816.24, p=.05\right]$. These trends suggested that subjects reading the homeschool story engaged in more critical scrutiny than did subjects reading the away-school story; similarly, HNC subjects tended to engage in more scrutiny than LNC subjects. However, the differences in the levels of scrutiny applied to a text were not accompanied by corresponding levels of belief change.

\section{EXPERIMENT 3}

One additional reason for the repeated failures to replicate the story effect may be that the weakly supported arguments used in the Prentice et al. (1997) stories for Yale and Princeton subjects were not perceived to be weakly supported by the Ohio State subjects. If the arguments were perceived as strong, increased scrutiny should have resulted in even greater levels of belief change (Petty \& Cacioppo, 1986). Thus, if the narrative passages contained a mixture of weak and strong arguments as perceived by the subjects, inconclusive effects for story setting and NC could have occurred. Therefore, in conjunction with a final replication attempt, Experiment 3, we conducted a supplementary study to verify the perceived weakness of story assertions. We were thus able to perform additional analyses based on a subset of verified weak assertions. 


\section{Method}

Subjects. Ohio State students $(N=201)$ participated in Experiment 3 for partial course credit. Sixty-four individuals read the control story, 68 read one of the OSU stories, and 69 read one of the Yale stories.

Design and Procedure. The design and procedure were identical to that of Experiment 2 with one exception. Due to time constraints, all four versions of the experimental stories were shortened by removing all true-story assertions, leaving only false assertions.

\section{Results and Discussion}

As with the previous two replications, an ANOVA of the means indicated persuasion by the narrative irrespective of story settings (see the bottom panel of Table 1). Relative to control subjects, experimental subjects demonstrated greater agreement with false test items than they did with true test items $\left[F(1,132)=51.37, M S_{\mathrm{e}}=1.91\right.$, $p<.001]$. Thus, subjects were persuaded by the false-story assertions. This effect was not moderated by story location $\left[F(1,132)=.68, M S_{\mathrm{e}}=1.91, p<.45\right]$. The two-way interactions for both the OSU $\left[F(1,66)=58.61, M S_{\mathrm{e}}=\right.$ $1.91, p<.001]$ and Yale $\left[F(1,66)=19.80, M S_{\mathrm{e}}=1.91\right.$, $p<.001]$ story-location conditions attained significance. Our inability to repeat the story-setting effect, as measured by average persuasion in the direction of story assertions, is shown in the rightmost graph of Figure 1. Additionally, there were no main effects or interactions involving $\mathrm{NC}$ (all $p \mathrm{~s}>.25$; median $\mathrm{NC}=58$, range $=18-88$ ).

The Pinocchio procedure again provided some support for differential scrutiny as a function of story location and NC. Subjects reading the home-school story showed nonreliable trends to identify more false-sounding text $(M \mathrm{~s}=24.1$ for circles and 38.8 for lines) than did individuals reading the away-school story $(M \mathrm{~s}=16.9$ and 31.4 , respectively). HNC subjects identified more suspicious lines in the text than did LNC subjects $[M=38.9$ vs. $\left.M=32.0 ; F(1,122)=3.92, M S_{\mathrm{e}}=359.54, p=.05\right]$, although the effect for circles did not attain statistical significance. As in Experiment 2, these results suggested some effect of story setting and NC on story scrutiny; however, there were no parallel effects on the dependent measures of belief change.

In a supplementary study undertaken to limit analyses to verified weak assertions, 59 OSU students completed booklets which contained false assertions from the experimental story as well as four arguments that previous pretesting had established as weak (see Petty \& Cacioppo, 1986). These pretested arguments were included to ensure that the present supplementary sample replicated previous argument-strength testing results, and would therefore provide accurate estimates of the strengths of experimental story assertions (Wheeler, 1997).

After reading each argument, subjects were given the opportunity to record the thoughts and feelings they had had while reading the passage. Using a standard thoughtlisting procedure (Brock, 1967), weak arguments were defined as ones that elicited a greater proportion of negative to positive thoughts (Petty \& Cacioppo, 1986). The two belief-change indices were recomputed so as to contain only the subset of 10 story assertions that were established as weak by this criterion. The same ANOVA tests used above were then applied to these new dependent measures.

Elimination of the nonweak story items made little difference. A significant test-item effect was obtained again $\left[F(1,132)=44.50, M S_{\mathrm{e}}=3.25, p<.001\right]$, but there was no evidence for moderation of belief change by story setting $\left[F(1,132)=.76, M S_{\mathrm{e}}=3.25, p>.35\right]$ or by NC [fourway $\left.F(1,132)=.21, M S_{\mathrm{e}}=3.25, p>.60\right]$.

\section{GENERAL DISCUSSION}

Using the materials and measures of Prentice et al. (1997), we examined the impact of story setting and NC on narrative-based persuasion. Across all three replications, we found that individuals were persuaded by story assertions. The story assertions reflected topics about which the subjects presumably had prior contrary knowledge (e.g., "A college education will hurt your chances of getting a good job"). The Prentice et al. experiments and our replications constitute strong evidence for the persuasiveness of narratives. Most importantly, the belief changes we observed were not reliably moderated by the setting of the story (Figure 1). In contrast to the null home-location effect reported by Prentice et al. (1997), our analyses indicated that subjects were persuaded by both the remote and local story versions. Today, as in the past, a parable by Jesus would not be discounted because it reflected local settings and issues.

We earlier noted that personal relevance (Petty \& Cacioppo, 1986) had been manipulated by use of messages that pertain to a familiar setting (own college) versus to an unfamiliar setting (another college). However, despite the fact that the present instantiations of personal relevance were standard operations, relevance differences did not have corresponding effects on belief differences.

Is the original story-setting effect (Prentice et al., 1997) attributable to sampling error or, alternatively, have we failed to locate the factor that could resolve discrepancies between our results and those of Prentice et al.?

One possibility was that subjects at Yale and Princeton were "smarter" than the OSU subjects overall and that intelligence would therefore moderate the story-location effect. Perhaps only highly intelligent individuals reject story assertions when reading a story that takes place at their own school. However, in an analysis combining our three experiments and using our SAT questionnaire as a proxy for intelligence, intelligence failed to interact with story location (Wheeler, 1997). In fact, the effects found for SAT were in direct contradiction to the above hypothesis: more "intelligent" individuals showed greater levels of persuasion by the story assertions overall (Wheeler, 1997). Thus, differential intelligence seemed unable to account for the present pattern of results.

A second possible explanation was that individuals at Yale or Princeton may have been more likely to engage in effortful thinking than individuals at OSU. The entire NC distribution for our samples may have been lower than those at Princeton and Yale, and thus median splits may not have been sufficient to capture these differences. 
However, in analyses that collapsed across experiments and used only the upper and lower quartiles of NC subjects (Wheeler, 1997), we still failed to find moderation of the story-location effect by NC.

Third, perhaps our manipulation of story setting entailed a confound. The manipulation of story distance may have concurrently been a manipulation of communicator prestige or credibility. Consider that the Yale story may have more prestigious connotations than the Ohio State story because of the high academic standards typically associated with Yale. It is possible that this confound obscured our results, but, in fact, this confound should have made the Prentice et al. (1997) pattern easier to obtain. If the prestige or credibility of the Yale communicators was higher, subjects should have been more persuaded by the Yale story. However, even in the face of this "supportive" confound, we failed to find persuasion differences between the two stories.

Fourth, the present Pinocchio procedure provided tentative support for the claim by Prentice et al. (1997) that individuals scrutinize the assertions in a fictional narrative more when the setting is local rather than remote. Subjects reading the home-school story tended to indicate that they had found more portions of the narrative dubious than had subjects reading the away-school story. However, despite the somewhat higher levels of scrutiny by the subjects reading the home-school story, their beliefs did not differ from those of subjects reading the awayschool story.

Finally, we considered whether the assertions provided in the story were unambiguously weak. While the assertions were presented in a manner that should preclude belief change in the presence of scrutiny, these assertions may have been perceived as stronger by the Ohio State subjects than by the Yale or Princeton subjects. A supplementary study of weak assertions (in conjunction with Experiment 3) showed that the predicted story-location effect could not be obtained for verifiably weak assertions.

Despite the rhetorical style of the assertions used in our materials, elaboration, as defined in the mainstream attitudes literature, may not adequately account for narrative-based persuasion. A similar conclusion was recently reached by Prentice and Gerrig (in press):

We described several models of nonsystematic processing that may be useful for characterizing the way readers process fiction. In particular, models of heuristic processing (Chaiken, 1987) and peripheral route processing (Petty \& Cacioppo, 1986) ... . We have been hesitant to embrace either of these models, however, because neither of them seems to capture the phenomenological experience of reading (or hearing or viewing) a work of fiction.

Indeed, because readers may not expect or even perceive persuasive attempts in narratives, elaborative processing may not determine subsequent belief change. Narratives may involve many details (e.g., visual descriptions, extraneous character dialogue, etc.) that are tenuously related to any encompassed argumentation. As a result, any scrutiny may be sharply diluted while other processes drive belief change (Green \& Brock, 1996).

In conclusion, the results across the Prentice et al. (1997) experiments and our own replications best supported the proposal that disbelief must be constructed (Gilbert, 1991). The subjects in all five experiments, the two by Prentice et al. and the three presented here, accepted false assertions even when they were explicitly told that the story was fictional. Although, in the present replication attempts, cognitive scrutiny was affected by NC and story setting in theoretically predicted directions, such scrutiny did not influence differential construction of disbelief. If the story setting effect (Prentice et al., 1997) was indeed an anomalous result, the persuasive efficacy of assertions in fictional narratives is more general and more robust than hitherto suspected.

\section{REFERENCES}

Brock, T. C. (1967). Communication discrepancy and intent to persuade as determinants of counterargument production. Journal of $E x$ perimental Social Psychology, 3, 269-309.

Cacioppo, J. T., Petty, R. E., Feinstein, J. A., \& Jarvis, B. W. G. (1996). Dispositional differences in cognitive motivation: The life and times of individuals varying in need for cognition. Psychological Bulletin, 119, 197-253.

Cacioppo, J. T., Petty, R. E., \& KaO, C. F. (1984). The efficient assessment of need for cognition. Joumal of Personality Assessment, 48, 306-307.

Chaiken, S. (1987). The heuristic model of persuasion. In M. P. Zanna, J. M. Olson, \& C. P. Herman (Eds.), Social influence: The Ontario Symposium (Vol. 5, pp. 3-39). Hillsdale, NJ: Erlbaum.

EAGLY, A. H., \& ChaikEN, S. (1993). The psychology of attitudes. Fort Worth, TX: Harcourt Brace Jovanovich.

GiLbert, D. T. (1991). How mental systems believe. American Psychologist, 46, 107-119.

GREEN, M. C. (1996). Mechanisms of narrative-based belief change. Unpublished master's thesis, Ohio State University.

Green, M. C., \& Brock, T. C. (1996). Mechanisms of narrative persuasion. International Journal of Psychology, 31, 13-14.

PETTY, R. E., \& CACIOPPO, J. T. (1986). Communication and persuasion: Central and peripheral routes to attitude change. New York: Springer-Verlag.

Prentice, D. A., \& Gerrig, R. J. (in press). Exploring the boundaries between fiction and reality. In S. Chaiken \& Y. Trope (Eds.), Dual-process theories in social psychology. New York: Guilford.

Prentice, D. A., Gerrig, R. J., \& Bailis, D. S. (1997). What readers bring to the processing of fictional texts. Psychonomic Bulletin \& Review, 4, 416-420.

WHEELER, S. C. (1997). Structural and dispositional determinants of the persuasive impact of fiction. Unpublished master's thesis, Ohio State University.

\section{NOTES}

1. One of the original Prentice et al. (1997) assertions, regarding college admissions for children of alumni, was dropped from our design because it was not relevant to OSU students.

2. See Wheeler (1997) for details of all interactions involving NC for the present experiments.

(Manuscript received November 6, 1997; revision accepted for publication March 26, 1998.) 